\title{
FUNGAL INFECTIONS ASSOCIATED WITH COVID-19
}

\author{
ANTA SHARMA*, SONIKA TANWAR* ${ }^{*}$ RAJESH ASIJA*, RICHA AGARWAL* \\ ${ }^{*}$ Maharishi Arvind Institute of Pharmacy, Jaipur 302020, Rajasthan, India \\ *Email: richaagarwal509@gmail.com
}

Received: 27 Jun 2021, Revised and Accepted: 16 Aug 2021

\author{
ABSTRACT \\ To study fungal infections such as Mucormycosis, Aspergillosis, Candidiasis, Cryptococcosis associated with Covid-19.
}

A detailed study was done with the information gathered from the articles in specified databases, online sources, and online published materials to have current details of the situation of fungal infections in covid patients.

Fungal infections were seen among covid-19 patients mostly due to opportunistic fungal pathogens such as Mucor, Candida, Aspergillus, and Cryptococcus. The reason behind rising opportunistic fungal infections among covid-19 patients may be the immunocompromised host. The most common species responsible for fungal infections in covid-19 were noticed to be of genus Mucor, A. flavus, and A. fumigatus species of genus Aspergillus, C. albicans species of genus Candida, C. neoformans, and C. gattii species of genus Cryptococcus.

Patients suffering or recovered from covid-19 are now facing numerous Secondary Infections. The majority of secondary infections associated with covid-19 are Fungal Infections. Mucormycosis, candidiasis, aspergillosis, cryptococcosis as opportunistic infections are seen widely in the covid-19 treated patients. Rapid progression of such fungal infections is required to be controlled by early diagnosis of infection and by identifying the underlying risk parameters. Protocols for disease management will be beneficial too.

Keywords: Covid-19, SARS-CoV-2, Fungal infections, Mucormycosis, Candidiasis, Aspergillosis, Cryptococcosis

(c) 2021 The Authors. Published by Innovare Academic Sciences Pvt Ltd. This is an open-access article under the CC BY license (https://creativecommons.org/licenses/by/4.0/) DOI: https://dx.doi.org/10.22159/ijpps.2021v13i10.42554. Journal homepage: https://innovareacademics.in/journals/index.php/ijpps.

\section{INTRODUCTION}

The Global Popularity of covid-19 and the possibility of occurrence of Fungal Co-infections has become an emergency worldwide. Just like Severe Acute Respiratory Syndrome Coronavirus (SARS-CoV) and the Middle East Respiratory Syndrome Coronavirus (MERS-CoV), Severe Acute Respiratory Syndrome Coronavirus-2 (SARS-CoV-2) is in charge of lower respiratory infection and can cause Acute Respiratory Distress Syndromes (ARDS). Besides this, diffuse alveolar damage with severe inflammatory secretion, covid-19 patients always has immunosuppression with a decrease in CD4-T and CD8-T cells. Critically ill patients, especially the patients who are admitted to the intensive care unit (ICU) and require mechanical ventilation, or having a longer duration of hospital stay, are more likely to develop fungal coinfections. Hence, it is significant to observe that covid-19 patients can further develop fungal infections during the middle and latter stages of this disease, especially severely ill ones [1].

Fungi exist in two forms that are yeast and molds, former is typically a single, small and oval structure, whereas the latter consists of filamentous strands called hyphae. Fungi responsible for diseasecausing in healthy individuals are considered as a true pathogen, while those who develop the disease in immune-suppressed individuals are referred to as opportunistic pathogens, which mainly involve species of Mucor, Candida, Aspergillus, and Cryptococcus [2]. These four opportunistic fungal pathogens are reported to cause infections in covid-19 patients too [3]. This article is about the review of these pathogens, including the terms black, white, yellow, cream, and green fungus, which is the point of discussion nowadays.

\section{Search criteria}

The selections of articles for the current review were searched from specialized databases such as IAS-AJPCR, Elsevier, Pubmed, and Cambridge using the keywords. Other selections include articles from Springer, information from Internet sources, and Online published articles from The Lancet Respiratory Medicine, Medscape, and Statpearls. The Keywords utilized for searching include the term related to fungal infections and covid patients such as "post covid fungal infections" OR "fungal infections in patients recovered or suffering from covid-19". Articles displayed on databases were screened manually based on their title. Irrelevant pieces of information were excluded and the information on Internet sources that were up to date and resembled the present scope information was included in the study. Inclusion of study was based on criteria as-a) articles from peer-reviewed journals are to be included. b) Information from Internet sources should contain the present scope. c) Fungal infections that were detected in post covid patients (202021). Articles reviewed were only those which were written in English and published till now that is 2021 and the information from the internet included were those which resembles with study criteria, written in English and contains the latest information regarding the topic and filtered for 2020 and 2021. After screening and filtration 34 studies met the inclusion criteria and were included in the review. The selection strategy followed is portrayed in fig. 1.

\section{Mucor and mucormycosis}

Mucormycosis, among the many severe secondary infections associated with covid-19 have high mortality and rising incidence in affected and recovered patients [4]. Mucormycosis is the form of Zygormycosis caused by genus Mucor of Phylum Zygomycota which is described as a lethal infection mostly occurring in an immunocompromised host, patients with uncontrolled Diabetes mellitus, with leukemia and lymphoma $[5,6]$. Nomenclature of mucormycosis is done by its anatomic site localization instead of its Mycological Classification that is 1 of 6 forms: (1) Rhino cerebral (2) pulmonary (3) cutaneous (4) gastrointestinal (5) disseminated (6) uncommon presentations [7]. Fungi gain entry via inhalation into the paranasal sinuses and spread to the sphenoid sinus, palate, and cavernous sinus [6]. High chances of this infection are in those that are on hemodialysis, having a high dose of glucocorticoids, have any kind of trauma like burns, have acquired immunodeficiency syndrome, hematological malignancy, and those who have gone for organ transplantation [4-6]. A recent review says that $8 \%$ of covid Positive or recovered Patients had secondary bacterial or fungal infections during their hospital admission, and also due to the widespread use of broad-spectrum antibiotics and Steroids [4].

\section{Prevalence and incidence in India}

The incidence of mucormycosis as a post covid secondary infection reported from different parts of India till 25th May is shown in table 1. 


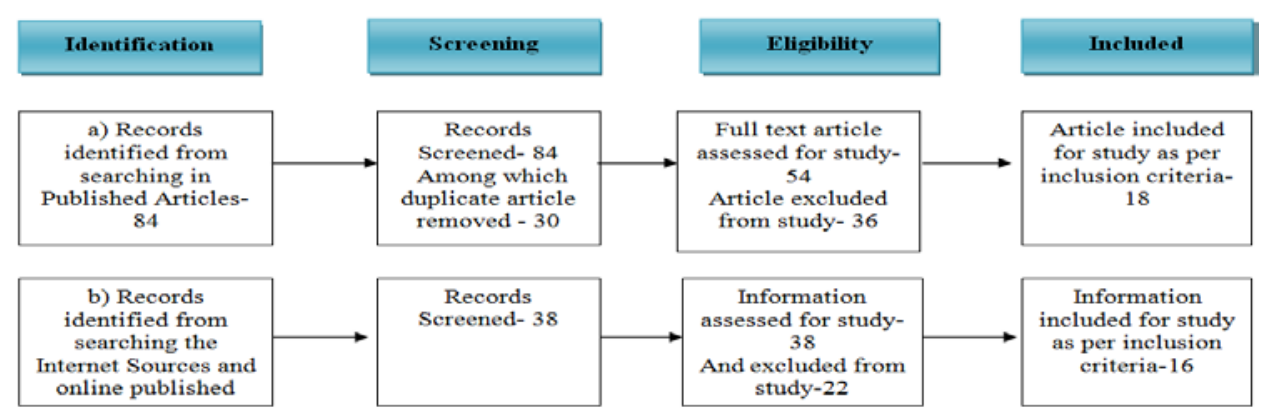

Fig. 1: Strategies followed for study selection

Table 1: The list of mucormycosis patients is as per the data shared by union minister of India, Sadananda gowda on May 25

\begin{tabular}{|c|c|c|c|c|c|}
\hline S. No. & State & Covid cases till 25th may & Mucormycetes cases till 25th & Ratio & Reference \\
\hline 1 & Andhra Pradesh & 1593821 & 768 & 1593821:768 & {$[8,9]$} \\
\hline 2 & Bihar & 692420 & 215 & $138484: 43$ & {$[8,9]$} \\
\hline 3 & Chandigarh & 58734 & 83 & $58734: 83$ & {$[8,9]$} \\
\hline 4 & Chhattisgarh & 953209 & 103 & 953209:103 & {$[8,9]$} \\
\hline 5 & Delhi & 1418418 & 119 & 1418418:119 & {$[8,9]$} \\
\hline 6 & Goa & 147861 & 10 & $147861: 10$ & {$[8,9]$} \\
\hline 7 & Gujarat & 791657 & 2859 & 791657:2859 & {$[8,9]$} \\
\hline 8 & Haryana & 741785 & 436 & $741785: 436$ & {$[8,9]$} \\
\hline 9 & Himachal Pradesh & 180983 & 3 & 180983:3 & {$[8,9]$} \\
\hline 10 & $\mathrm{~J}$ and $\mathrm{K}$ & 272858 & 5 & 272858:5 & {$[8,9]$} \\
\hline 11 & Jharkhand & 331811 & 29 & $331811: 29$ & {$[8,9]$} \\
\hline 12 & Karnataka & $2,450,215$ & 481 & $2450215: 481$ & {$[8,9]$} \\
\hline 13 & Kerala & 2365787 & 36 & $2365787: 36$ & {$[8,9]$} \\
\hline 14 & Madhya Pradesh & 767274 & 752 & $383637: 376$ & {$[8,9]$} \\
\hline 15 & Maharashtra & 5602019 & 2770 & $5602019: 2770$ & {$[8,9]$} \\
\hline 16 & Odisha & 703441 & 15 & $703441: 15$ & {$[8,9]$} \\
\hline 17 & Pondicherry & 96982 & 2 & 48491:1 & {$[8,9]$} \\
\hline 18 & Punjab & 543475 & 141 & 543475:141 & {$[8,9]$} \\
\hline 19 & Rajasthan & 920456 & 492 & $230114: 123$ & {$[8,9]$} \\
\hline 20 & Tamil Nadu & 1877211 & 236 & $1877211: 236$ & {$[8,9]$} \\
\hline 21 & Telangana & 556320 & 744 & $23180: 31$ & {$[8,9]$} \\
\hline 22 & Tripura & 47298 & 1 & 47298:1 & {$[8,9]$} \\
\hline 23 & Uttar Pradesh & 1673785 & 701 & $1673785: 701$ & {$[8,9]$} \\
\hline 24 & Uttarakhand & 315590 & 124 & $157795: 62$ & {$[8,9]$} \\
\hline 27 & Total & 25103410 & $\begin{array}{l}11125 \\
\text { The Average ratio is 5020682:2225 }\end{array}$ & 5020682:2225 & {$[8,9]$} \\
\hline
\end{tabular}

The table clarifies that on average of every 50, 20,682 covid infected persons, there are 2225 Mucor patients. Even the new reported Mucor cases are less in number. India still contributes to $45 \%$ of the new globally reported cases and about $34 \%$ of deaths during the third week of May 2021 [10].

\section{Underlying diseases and risk factors}

The major underlying disease being the risk factor for Mucor infection is Diabetes mellitus, followed by Leukemia and organ transplantation. However, the alarming threat for the Indian population is mucormycosis in an immunocompromised host [11]. Several studies claim that the patients on hemodialysis, having a high dose of Glucocorticoids, having low phagocyte count, impaired phagocyte function, neutropenia are more susceptible to Invasive Mucormycosis [6].

A study claims that in patients with poorly controlled Diabetes mellitus, elevated blood glucose levels lead to impaired neutrophil function. In Ketoacidosis, the hyperglycemia and acidic $\mathrm{pH}$ may be expected to result in a defect in the motility and killing activity of neutrophils. As the $\mathrm{pH}$ becomes acidic, the iron-protein complexes dissociate, which allows for the fungal cells to use the increased free iron and initiate growth [6].

Furthermore, as covid-19 is life-threatening, affected patients show overexpression of inflammatory cytokines and impaired cellmediated immunity with decreased $\mathrm{T}$ helper cell counts, which indicate susceptibility to fungal co-infections [4].
Critically ill patients, like those in intensive care units and those who require mechanical ventilation or who had longer hospital stays are more likely to develop fungal co-infections. Larger steroids use for covid management can also suppress the immunity of the patients, which makes them more prone to opportunistic fungal infections [4].

\section{Symptoms of mucormycosis}

- Pain and redness around eyes and/or nose

- Fever

- Numbness or swelling

- Headache

- Coughing

- Shortness of breath

- Bloody vomits

- Altered mental status

- Sinusitis-nasal

blockage or congestion

- Nasal discharge

(blackish/bloody)

- Local pain on the

cheekbone

- One-sided facial pain
- Blackish discoloration over the bridge of nose/palate Toothache

- Loosening of teeth

- Jaw involvement

- Blurred or double vision with pain

- Skin lesion

- Thrombosis and necrosis (eschar)

- Chest pain

- Pleural effusion

- Haemoptysis

- Worsening of respiratory symptoms [12] 


\section{Treatment and outcomes}

Diagnosis is dependent upon clinical features, pathological findings, and imaging to define the extent of involvement. Early diagnosis and timely surgery facilitate disease control. The primary guideline for treatment is to correct the responsible cause but it is not possible in patients reliant on high dose steroid therapy like in covid-19 [5].

The two mainstays of treatment are medical treatment with amphotericin B and with surgical debridement [5]. Be waiting for cultures delays the treatment process; direct positive smears may become sufficient for treatment initiation. Hyperbaric oxygen therapy and treatment with amphotericin B are adjunct modalities. Amphotericin B is fungistatic rather than fungicidal which increases its treatment duration [5]. Monitoring of kidney function is important due to abnormal nephrotoxic incidence of amphotericin B. second-line therapies may be contemplated in case of extensive disease [6].

Recommended second-line therapy is with echinocandins and amphotericin B. Echinocandins in combination with amphotericin B add a polyene backbone and the success of therapy increases. Some other second-line drugs include triazoles, posaconazole, and isavuconazole. Triazoles inhibit the 14- $\alpha$-demethylation, which causes an increase in toxic $14-\alpha-$-methyl sterols which then alters the fungal membrane's permeability [6].

Patients that are intolerant to amphotericin $\mathrm{B}$ are given posaconazole. Isavuconazole is used in the treatment of Invasive Mucormycosis because of its extended spectrum. And thus, it is the only antifungal used in the treatment of Invasive Mucormycosis [6]. Prognosis depends on multiple factors prompt initiation of treatment is an essential element [5].

After the confirmation of diagnosis, conservative management is initiated for the patient [5]. Identification and treatment of any underlying condition help in assuring a fair prognosis [6]. It is important to take care of hyperglycemia and ketoacidosis in uncontrolled Diabetes mellitus patients. Additionally, tapering immunosuppressive therapy plays an additional role in the treatment of mucormycosis [6].

\section{Aspergillus and aspergillosis}

Co-fungal infections are occurring post-covid-19 and it is reported that different species of the following genera are causing it: Candida, Cryptococcus, Mucor, and Aspergillus [3]. Aspergillus is one of the most common pathogens which cause deadly fungal infections. Infection is typically caused by Aspergillus fumigatus (about 90\%), Aspergillus flavus, Aspergillus nidulans, Aspergillus niger, Aspergillus terreus, and Aspergillus versicolor. Nowadays, non-fumigatus pathogens have also become frequent causative agents of this. Identification of the precise species is important as there are variations in antifungal susceptibility profiles of different species, and also there are differences in how the different species produce their clinical presentations [13]. Various species of Aspergillus are present in the environment and most commonly, Aspergillus fumigatus is present. They cause a wide range of infections in humans like Invasive Pulmonary Aspergillosis (IPA), Chronic Pulmonary Aspergillosis (CPA), Allergic Broncho-Pulmonary Aspergillosis (ABPA), chronic rhinosinusitis, fungal asthma, and Aspergillus bronchitis, etc. Not only this it has been found that up to $40 \%$ of covid-19 hospitalized patients can develop severe ARDS, and therefore become more susceptible to acquire co-infections caused by bacteria, viruses, or fungi [14]. Patients with clinical symptoms of covid-19, reverse transcription-polymerase chain reaction (RT-PCR) test confirmed positive, and those requiring intensive care due to developing respiratory insufficiency are at high risk for Covid Associated Pulmonary Aspergillosis (CAPA). The entry criterion proposed was this: positive RT-PCR for SARS CoV-2 anytime during $2 \mathrm{w}$ between hospital admission and ICU admission and or positive RT-PCR within 72-96 h after ICU admission. CAPA might develop during the following weeks, and the risk could be further increased for superinfections by anti-IL-6-receptor treatment for covid-19 or corticosteroid treatment for underlying conditions [14].

\section{Association between covid-19 and aspergillosis}

The role of interleukin-10: IL-10 regulates cellular immune responses and is therefore involved in various inflammatory diseases. The highly elevated level of serum IL- 6 and IL-10 can lead to disease progression as increased production of IL-10, mediates the influx of phagocytic cells, which can limit the extent of local tissue destruction of Aspergillus infection. Greater Th-2 (T helper type 2) cells responses that are the increase of IL-10 or lesser Th1 (T helper type 1) cells responses, down-regulate macrophage responses, and increase host susceptibility to deadly Aspergillus infection [3].

The role of interleukin-6: pro-inflammatory cytokines and chemokines which are elevated in severe covid-19 patients are TNFa, IL-6, IL-10, interleukin-1b, and monocyte chemoattractant protein-1. Elevated cytokine levels can lead to lethal complications of covid-19. Tissue necrosis and interstitial infiltration with macrophage and monocyte in the lung, heart, and gastrointestinal mucosa are found in severe covid-19 patients with elevated inflammatory cytokines. IL- 6 is part of the important cytokines among the excessive Cytokines Releasing syndrome (CRS). IL-6 is a multi-functional cytokine and it plays an important part in protective immunity against Aspergillus. After Aspergillus fumigatus infection, there is a significant increase in the amount of IL-6. Reduced responsiveness is exhibited of T cells to IL- 6 in patients with IPA. Covid-19 patients with CRS show excessive IL-6 signaling which leads to numerous biological effects such as increasing vessel permeability, ARDS, cardiac arrhythmia, and reducing myocardial contractility. The patients more vulnerable to Invasive Pulmonary Aspergillosis are the non-immune compromised patients with ARDS whose prevalence can reach up to $15 \%$ of patients. Beneficial additions to antiviral therapy are immune modulators. IL- 6 blockade targets the host immune system that can be effective for covid- 19 . The drug tocilizumab is a recombinant humanized monoclonal antiIL-6 receptor antibody. Tocilizumab is hereby approved for patients with covid-19 pneumonia, ARDS, and elevated IL-6. There should be an early diagnosis of Cytokines Releasing syndrome in covid-19 patients and not only this. There should be prompt initiation of immune-modulatory treatment. The duly intervention of elevated serum IL-6 levels in patients can avoid the progression and complications of covid-19. But IL- 6 can be a double-edged sword, tocilizumab can be applied in the treatment of covid-19 as an antiIL-6 agent, but it can also potentially cause Aspergillus infection by decreasing IL-6 immune response [3].

\section{Diagnosis}

Diagnosis of CAPA includes the following:

1. Galactomannan (GM) detection from Serum/Bronchoalveolar Lavage Fluid (BALF)/Endotracheal Aspirates (ETA).

2. Isolation of Aspergillus species from BALF/ETA/sputa

\section{Serum Beta-d Glucan (BDG) detection.}

4. Detection of Aspergillus Deoxyribonucleic Acid (DNA) by real-time Polymerase Chain Reaction (PCR) in blood or respiratory samples.

This approach may aid in the early institution of antifungal therapy [15].

\section{Risk factors}

Significantly, the pathogenesis of IPA differs between neutropenic and non-neutropenic patients and those with covid-19, impacting clinical presentation, radiological findings, and diagnostic test results in the mycology laboratory. Despite these significant differences, revised European Organization for Research and Treatment of Cancer/Invasive Fungal Infections Cooperative Group and National Institute of Allergy and Infectious Diseases Mycoses Study Group (EORTC/MSG) definitions focus mainly on neutropenic patients with underlying hematological malignancies and "typical" presentation of IPA and have limited applicability and inferior performance in non-neutropenic patients who frequently do not cover radiological and host criteria, including patients with covid-19. Therefore an alternative clinical algorithm is created for diagnosing IPA in the ICU setting, which defines putative IPA and is now the 
standard of care for defining IPA in the ICU, but still, highly reliable definitions of IA (Invasive Aspergillosis) are missing (as work on improved definitions is still in progress).

The Rapid development of CAPA a few days after ICU admission matches the observation made for influenza-associated pulmonary aspergillosis. Risk factors vulnerable to covid-19 patients to develop secondary pulmonary aspergillosis are the same as those identified for influenza-IPA superinfections. The main risk factors include severe lung damage during covid-19, the use of corticosteroids in those with ARDS, the widespread use of broad-spectrum antibiotics in ICUs, and the presence of comorbidities such as structural lung defects [16].

Pulmonary fibrosis can be caused by the cytokine storm activated by the viral antigens, harmful effects posed by drugs, high airway pressure, and hypoxia-induced acute lung injury secondary to mechanical ventilation. While interstitial pulmonary fibrosis per se does not lay open to the development of IPA, a small group of these covid-19 survivors may require corticosteroid treatment for a long time, which may lay them open to CAPA years after the acute phase of the viral infection. In cases with ARDS, systemic corticosteroids are used to alleviate the immune responses and prevent cytokine storms, but they may predispose to developing secondary infections at the same time [16].

Although there is no detailed information on antibiotic use among patients, broad-spectrum antibiotics are assumed to be used in $75 \%$ of covid-19 patients admitted to ICU. As the human gut microbiome is a highly complicated structure of bacteria and fungi, and even the bacteria are the most diverse constituents, the intake of antibiotics results in the disturbance of microbiome steady-state composition, which allows fungi to flourish, and may make the host vulnerable to destroying fungal infections once the immune system is impaired [16].

Underlying medical conditions may also cause covid-19 patients to develop CAPA. Hypertension (17/35; 49\%), diabetes (9/35; 26\%), obesity $(8 / 35 ; 23 \%)$, Chronic Obstructive Pulmonary Disease (COPD) $(5 / 35 ; 14 \%)$, heart diseases $(5 / 35 ; 14 \%)$, hypercholesterinemia $(4 / 35 ; 11 \%)$, and asthma $(3 / 35 ; 9 \%)$ are the most prevalent comorbidities found out yet. While diseases like hypertension, coronary heart diseases, and diabetes increase the risk of infection. Overall the structural lung damage caused by COPD or asthma can particularly put patients at risk to develop IPA [16].

\section{Antifungal treatment strategies}

The clinical impression, or a proven diagnosis, of Aspergillus coinfection, should initiate empiric or targeted antifungal therapy, respectively, even though its effectiveness is not established. Many cases survived their infection and those dying lead to multi-organ failure and sepsis. International treatment guidelines suggest triazoles voriconazole or isavuconazole as the first-line treatment of IA. The emergence of multi-triazole resistance in A. fumigatus challenges the effectiveness of triazoles in the successful treatment of IPA, specifically in areas of high occurrence, and their use in such cases is associated with an increased death rate. Triazole hostility in A. fumigatus is causally linked to the use of triazole compounds that are structurally similar to those used in medical practice, as agricultural fungicides, or less commonly to long-term triazole use in individual patients. The early process of resistance typically affects azole näive patients and is distinguished by increased Minimum Inhibitory Concentrations (MIC) of itraconazole, voriconazole, posaconazole, and isavuconazole. These emphasize the significance of Antifungal Susceptibility Testing (AFST) either through phenotypic or genotypic methods to determine triazole hostility, which will help direct the choice of treatment. Although the cultures are well known to have a poor diagnostic susceptibility, the ability to culture Aspergillus species will allow for the establishment of MICs for triazoles. Recently, a four-well triazole resistance screening plate was confirmed for A. fumigatus, which can be useful in laboratories that cannot perform the suggested broth microdilution methods for AFST. Genotypic testing that uses molecular assays has also been tested to detect Aspergillus species and the common mutations linked with triazole hostility directly from clinical samples, which will allow for the rapid detection of a marker of hostility and guide treatment options. Many patients received a triazole-based AFT regimen either alone or in combination with an echinocandin or liposomal amphotericin B. Only a few cases reported sensitivity results based on either phenotypic testing and/or the detection of common mutations associated with triazole resistance using molecular techniques. Others have been reported as being caused by the resistance of Aspergillus fumigatus to triazole. All of which were confirmed to have the cyp51ATR34L98H mutation. Knowledge of the local epidemiology of triazole resistance is significant to help guide the choice of therapy while awaiting sensitivity results. It has been suggested that for areas with triazole hostility rates of $>10 \%$, voriconazole-echinocandin combination therapy or liposomal amphotericin B should be used as the initial therapy. However, in many countries, there are no inspection systems in place to detect the occurrence of triazole hostility in A. fumigatus, which is known to be the most common Aspergillus species causing IA, as it has also been noted in the CAPA cases reported to date [15].

It was reported that prophylactic aerosolized liposomal amphotericin$B$ was administered to all covid-19 patients on mechanical ventilation in critical care after they found a group of CAPA cases, among which few were proven. Antifungal prophylaxis formed part of the multifaceted management of this cluster, which also included the GM screening of serum and BALF twice a week if and when a bronchoscopy was performed. HEPA filters were also installed in their critical care unit. The logic for prospective trials is required to be determined to accept whether antifungal prophylaxis in severe covid19 cases is indicated. A clinical trial of posaconazole prophylaxis for the avoidance of pulmonary aspergillosis in patients with severe influenza (NCT03378479) is currently ongoing and this will give data on the efficacy of this approach at least for influenza [15].

New antifungal agents with a novel mechanism of action are in the pipeline to deal with the problem of antifungal hostility, which endangers the efficacy of the few agents currently being used to cure invasive fungal disease. Clinical trials are ongoing for new antifungal agents, namely, ibrexafungerp (NCT03672292), olorofim (NCT03583164), and fosmanogepix (NCT04240886). Ibrexafungerp, which is structurally the same as echinocandins, hinder fungal-1,3-glucan synthase with activity against triazole-resistant Aspergillus sp. Olorofim and fosmanogepix have different novel targets, which are fungal dihydroorotate dehydrogenase, a significant enzyme in the fungal DNA synthesis, and the obstruction of fungal enzyme Gwt1 inactivating change of mannoproteins, which is a significant component in maintaining fungal cell wall integrity, respectively. All three agents have activity against Aspergillus species, including A. fumigatus, which may impact the future management of patients with IA positively, specially CAPA [15].

\section{Candida and candidiasis}

As covid-19 is still at a peak in some countries, secondary infections are rising too. The most fatal and superior of those are fungal infections. A frequent type of superficially occurring fungal infection among them is candidiasis [17]. Species of yeast that belong to the Candida genus include Candida albicans, Candida glabrata, Candida parapsilosis, Candida tropicalis, and Candida krusei, which are the most widespread fungal species inhabiting mucosal surfaces like skin, respiratory mucosa, digestive and urinary tracts too [18].

Candida species are communion with humans and have virulence attributes too, enabling on which cause various infections in humans, especially when the immune system of the host is impaired [18]. Superficial infections associated with candidiasis are skin disorders, mucosal infections, Oropharyngeal or Vulvovaginitis Candidiasis, and Invasive Candidiasis [18]. Deep fungal infections occur by the proliferation of the pathogen in the deeper tissue area and are usually associated with ulceration and pricking of bony areas [17]. A patient with candidiasis has white patches/lesions mainly in the oral cavity (tongue, inner cheeks, and palate) said by experts from the national task force on covid-19, they further added that terming the candidiasis infection as the white fungus is incorrect and creating a link between them is a misnomer [19].

\section{Underlying diseases and cause}

\section{Etiology}

Candidiasis is an opportunistic infection. Candida albicans, present in healthy persons, colonizes the oropharyngeal, esophageal, and 
gastrointestinal mucosa. In an immunocompromised host, this species can cause mucosal candidiasis in the areas where they are usually present [18].

Patients with leukemia, lymphoma consumes corticosteroids and cytotoxic drugs which compromise their immunity and leading to candidiasis infection. Cancer chemotherapy may result in fungemia occurring due to Candida albicans, which develop from fungal translocation via compromised mucosal barriers [18]. Any changes in the host's environment and the size or composition of endogenous bacterial population fungal infection of the upper and lower GIT transform into an opportunistic pathogen [18].

Fungal colonization in the vagina increases in Diabetes mellitus, pregnancy, and the use of oral contraceptives. Oral candidiasis is mostly associated with HIV patients, more than $90 \%$ of HIV patients show candidiasis. Others are TB, myxedema, hypoparathyroidism, Addison's disease, nutritional deficiency (vitamin A, B6, Iron), smoking, poorly maintained dentures, IV tubes, catheters, heart valves, old age, infancy, and pregnancy, Xerostomia too due to the absence of protective antifungal proteins, histatin, and calprotectin [18].

\section{Epidemiology}

Infections related to Candida species have notably increased over the past few decades. Among this, one-third of invasive Candida infection is caused due to C. albicans [20]. Non-C. albicans species such as Candida glabrata (C. glabrata), Candida tropicalis ( $C$. tropicalis), Candida parapsilosis (C. parapsilosis), and Candida krusei (C. krusei) have emerged now as secondary pathogen as pathogenic candidiasis in neonates [21].

Among the Candida species, C. albicans was the most common species, followed by C. lusitaniae, C. parapsilosis, C. glabrata, C. kefyr, C. famata, C. africana, and C. orthopsilosis, respectively [18].

\section{Pathophysiology}

When normal host immunity is disturbed, Candida albicans cause thrush. The Proliferation of organisms in oral mucosa causes epithelial cell desquamation and accumulation of keratin, bacteria, and necrotic tissue. This debris forms a pseudomembrane that closely adheres to the mucosa. This pseudomembrane may rarely involve extensive areas of edema, ulceration, and necrosis of the underlying mucosa [18].

Candida reaches the bloodstream by three major routes. The most frequent of these is via the GIT mucosal barrier, others through an intravascular catheter and form a localized infection. Candida can pass into the bloodstream in neutropenia patients as well as in intensive care unit patients too. Candida is also a part of the normal gut microflora, so any condition which makes a person immunocompromised can cause Invasive Candidiasis in the bloodstream. Candida growth in indwelling catheters in central lines, can occur either at the implantation site or at the hub and cause prevalence to the next Candida infection. Localized infection may not cause bloodstream invasion, but ascending Candida urinary tract infection associated with intrinsic obstruction or extrinsic compression may cause bloodstream invasion [18].

Vulvovaginal Candidiasis may occur with the use of local or systemic antimicrobial therapy and may cause precipitate recurrent episodes of the disease. Hypothetically, the pathophysiology of Vulvovaginal Candidiasis may be due to reduction or change in routine vaginal flora, yeast colonization, and proliferation [18].

\section{Signs and symptoms}

Signs and symptoms of candidiasis vary depending on the area affected [22].

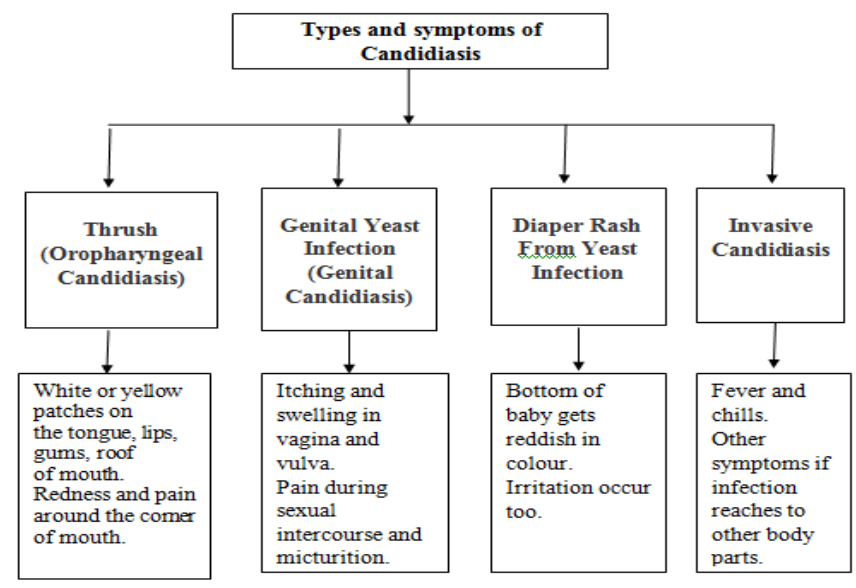

Fig. 2: Explaining types of candidiasis and their symptoms

\section{Treatment and management}

The management of Invasive Candidiasis in both, covid-19 patients and the non-covid-19 patient is similar [21]. Patients with a localized burning sensation in the oral cavity are sometimes managed with an empiric anti candidiasis treatment. An empirical approach for oral candidiasis treatment is also preferred due to the increased cost of laboratory investigation of candidiasis. The culture investigation helps in quantifying candidiasis organisms and in differentiating between normal and increased levels of oral candidiasis infections [23]. Nystatin oral suspension for oral rinse is used in oral candidiasis, with guidance to patients to retain the suspension in the oral cavity before swallowing [23]. Oral applications of probiotics can be used as an adjuvant in the treatment of oral candidiasis [23]. Clotrimazole is used in Oropharyngeal Candidiasis treatment [23].

For Invasive Candidiasis, echinocandins are the choice of treatment, with fluconazole, Liposomal amphotericin B, voriconazole, posaconazole, and isavuconazole being the second line alternatives
[21]. Fluconazole should be avoided in the first trimester of pregnancy [20]. Mild or moderate genital Candida infection can be treated with antifungal vaginal creams [21]. For recurrent vaginal Candida infections, fluconazole is prescribed [18]. The same is used for oral thrush too, with oral lozenges as a substitute [18].

Systemic Candidiasis is treated with oral or intravenous antifungal medications; including caspofungin, fluconazole, and amphotericin B [18]. Flucystine can be used in the treatment of systemic candidiasis [23].

In denture-related candidiasis, the patient should be guided to microwave disinfection of acrylic-based dentures. This should be disinfected with chlorhexidine, hydrogen peroxide, and antifungal solutions [23].

\section{Cryptococcus and cryptococcosis}

Cryptococcosis is potentially a deadly fungal infection commonly caused by inhalation of Cryptococcus neoformans or Cryptococcus gattii spores. Cases of covid-19 having shortness of breath are 
mostly found to have associated pulmonary cryptococcosis. Serious cryptococcal infection occurs in immunocompromised patients but can also occur in immunocompetent adults [24]. A review study claims that the evolution of the HIV pandemic was an influence on the epidemiology of the cryptococcal disease. The same incidence of rising new infections that is prevalent in the covid era once at a peak in Africa during the HIV pandemic. Cryptococcosis lies at the third position of most common fungal infections after Candida and Aspergillus [25]. Earlier the link between fungal infection and covid19 was reported with Aspergillus species. But now, a few cases are available with co-infection with Cryptococcus too. Taking into account the fact that covid-19 causes ARDS, co-infection with Cryptococcus species nearly increases inflammation in the lung and the danger for poor results. Since corticosteroids are an important treatment in covid-19 with respiratory symptoms and hypoxemia, starting them in patients with cryptococcal infection should be strictly monitored for the chances of dissemination. An analyst should get cultures for Cryptococcus species from the blood and the central nervous system in covid-19 patients with pulmonary cryptococcosis and maintain the requirement for corticosteroids with the danger of cryptococcal dissemination [24].

covid-19 patients with human immunodeficiency virus (HIV) infection accompanied by CD 4 T-lymphocyte count 200 cells/lL, Allogeneic Hematopoietic Stem Cell Transplantation (Allo-HSCT), SOT, or other immune impaired are sensitive to cryptococcosis, which is mostly present as meningoencephalitis. Given the difficulties adjoining the diagnosis of cryptococcosis and identification of Cryptococcus species, including $C$. neoformans and $C$. gattii species, the diagnosis of cryptococcosis is normally based on a combination of clinical and laboratory authentication. The methods employed to authenticate the infection are culture, direct microscopy, histopathology, serology, and molecular detection. To detect cryptococcosis, a sample of cerebrospinal fluid (CSF) can be combined with India ink and observed under a microscope that the characteristic structure of Cryptococcus species. With narrow budding encapsulated yeast usually can be found. Samples for culture should be given to Sabouraud dextrose agar at 30 degrees Celsius for $7 \mathrm{~d}$, in aerobic conditions, and checked daily. Moreover, cultures from patients receiving systemic antifungal therapy may need a longer time to rise. Cryptococcus is visible as creamy mucoid colonies. Capsular polysaccharides of Cryptococcus can be defined and quantified from body fluids such as serum, CSF, BAL, or pathological tissue. Three formats of Cryptococcal Antigen (CrAg) identification tests are now available: the Latex Agglutination Test (LAT), the Enzyme-Linked Immunoassay (EIA), and the Lateral Flow Immunoassay (LFA). These methods are rapid, sensitive, and particular but have not been standardized for respiratory specimens such as BAL, pleural fluid, or sputum. Molecular detection of Cryptococcus is needed in particular situations where additional diagnostic tools have failed to authenticate the detection of cryptococcosis. These molecular methods include pan-fungal PCR, DNA sequencing for identification, multiplex PCR, isothermal amplification method, and probe-based microarrays. Once cryptococcosis is diagnosed, a lumbar puncture and cerebrospinal fluid (CSF) examination (including antigen) is suggested in all patients. Cryptococcus can spread into the central nervous system, causing cryptococcal meningitis. The treatment suggestions are as follows: (i) Induction phase for amphotericin B deoxycholate and flucytosine, followed by fluconazole; alternative options for fluconazole, flucytosine or amphotericin B deoxycholate, fluconazole. (ii) Consolidation phases for fluconazole. (iii) Maintenance (or secondary prophylaxis) phase for fluconazole [1].

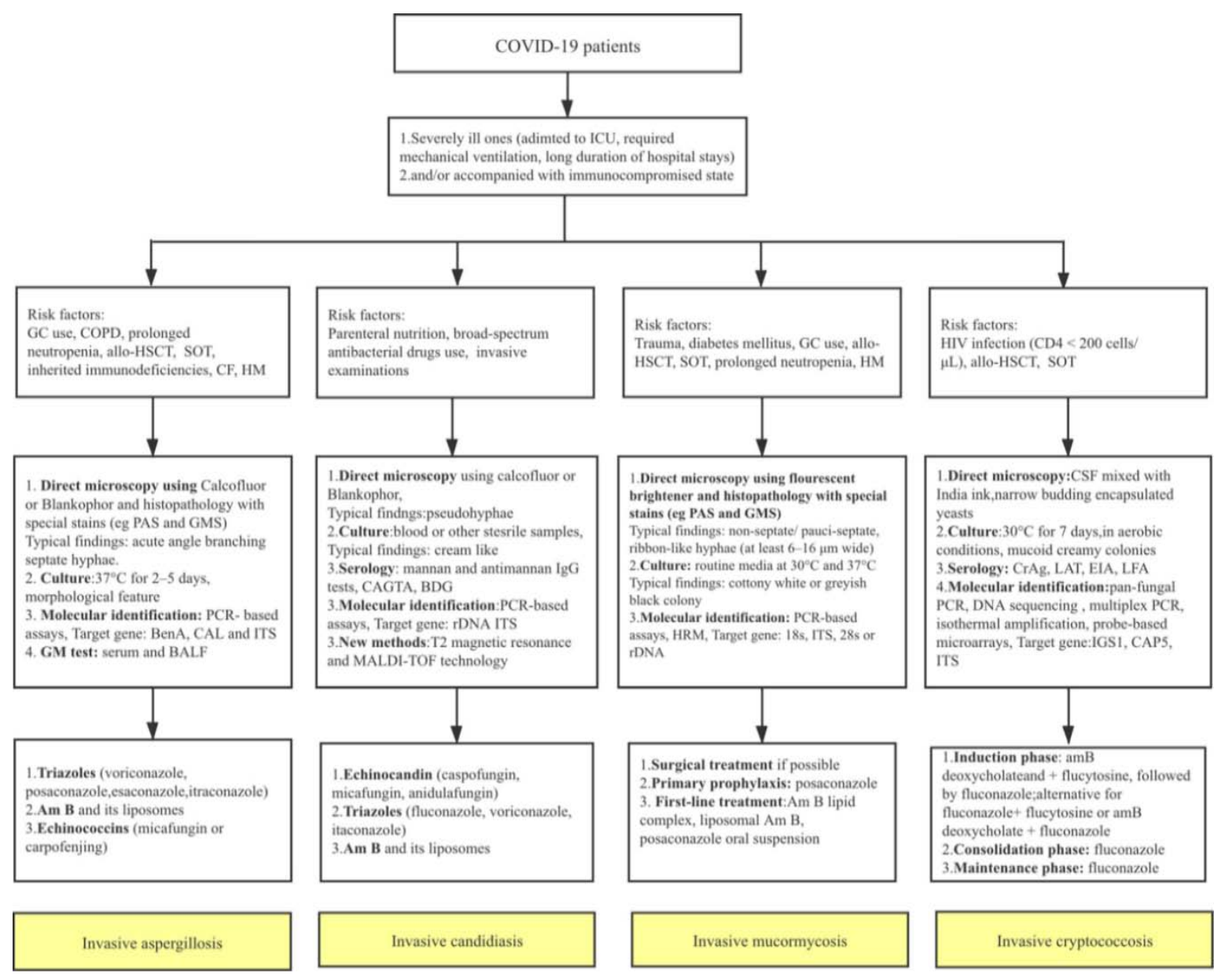

Fig. 3: Diagnostic and therapeutic pathways for invasive fungal co-infections [1] what are fungal infections, named by colors. Black, white, yellow, cream, and green? 


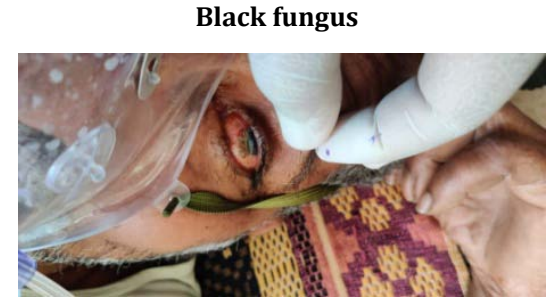

Fig. 4: Picture showing mucormycosis fungal infection in a person *Source-Acovid care center in Mandsaur, Madhya Pradesh, India

Black fungus is caused by the order mucorale, in which the genus implicated is Rhizopus, Mucor. Black fungus has given this term so as it causes the invasion of tissues and blood vessels and thus interrupts the blood supply to the normal tissue, which gets then necrosis and hence blackens the tissue [26].

\section{White fungus}

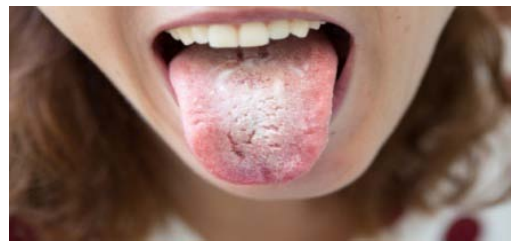

Fig. 5: Image shows the oral cavity affected by Candida albicans [27]

White fungus is caused by a yeast (a type of fungus) called Candida The most common species which cause the infection is Candida albicans [28]. White refers to the term Albicans. The white fungus has given this term as it grows in the lab as white spots on plates of agar and appears as white spots on the mucosa of the oral cavity [26]. Also, when it attacks private parts, there is a white color discharge, thus the name given is a white fungus [29].

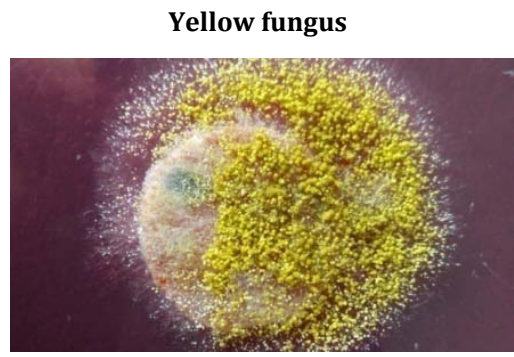

Fig. 6: Representational image of Aspergillus flavus [30]

Yellow fungus belongs to the Aspergillus family. Most likely, it is Aspergillus flavus from the Aspergillus family. Flavus is the Greek word for yellow [30]. The yellow fungus has been given the term because of the yellow color pus it forms in the wounded area of a patient infected with this fungus [30].

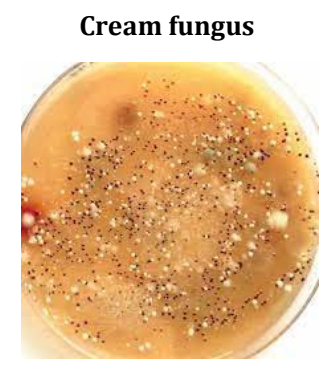

Fig. 7: Culture image of Cryptococcus gattii [31]
Cryptococcus species form creamy mucoid layers when cultured in the laboratory and hence the name cream fungus. The species of Cryptococcus which cause co-fungal infection with covid-19 are Cryptococcus neoformans and Cryptococcus gattii [1].

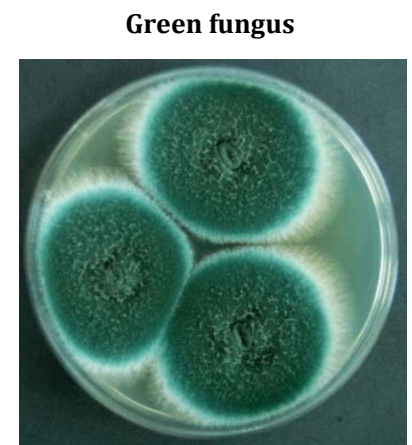

Fig. 8: Culture Image of Aspergillus fumigatus [32]

Green fungus is due to the Aspergillus family. The most prevalent cause of Aspergillus is Aspergillus fumigatus [33]. Green Fungus holds the term as the green color appears during its culture in the lab [34].

\section{CONCLUSION}

The global pandemic has affected every country up to an extent. Maintaining public health along with the economic condition is a difficult task for a country, but the rising of Secondary infections in this condition makes the situation tougher. Fungal infections as raised secondary covid infections have increased the mortality rate of covid patients. In India, the cases of secondary fungal infections are higher than in any other country. The reason behind increased fungal infections is still unknown, but several studies claim that the extensive use of steroids in covid patients has a key role in compromising the immunity of the host and making him susceptible to fungal infections. Others include hospital environment, ventilation state, longer hospital stay, underlying diseases like Diabetes mellitus, and renal failure, increases the risk of fungal infections. Each type of fungal infection has its management of therapy and symptoms so early diagnosis is necessary for the early management of the disease. Early prevention in susceptible patients will help in disease management. Protocols for disease management and therapy will be beneficial in the management of fungal infections.

\section{ACKNOWLEDGEMENT}

The authors are thankful to the faculty of Maharishi Arvind Institute of Pharmacy for guiding in the preparation of this manuscript.

\section{FUNDING}

Nil

\section{AUTHORS CONTRIBUTIONS}

All authors have equally participated in the designing and drafting of the article.

\section{CONFLICT OF INTERESTS}

Declared none

\section{REFERENCES}

1. Fungal co-infections associated with global COVID-19 pandemic: A clinical and diagnostic perspective from China. Mycopathologia. 2020 Jul:1-8.

2. Kaushik K, Agarwal S. The role of Herbal antifungal agents for the management of fungal diseases: A systemic review. Asian J Pharm Clin Res. 2019;12:34-40.

3. Lai CC, Yu WL. COVID-19 associated with pulmonary Aspergillosis: a literature review. J Microbiol Immunol Infect. 2021;54(1):46-53. doi: 10.1016/j.jmii.2020.09.004. 
4. Sharma S, Grover M, Bhargava S, Samdani S, Kataria T. Post coronavirus disease mucormycosis: a deadly addition to the pandemic spectrum. J Laryngol Otol. 2021;135(5):442-7. doi: $10.1017 /$ S0022215121000992.

5. Maini A, Tomar G, Khanna D, Kini Y, Mehta H, Bhagyasree V. Sino-orbital Mucormycosis in a COVID-19 patient: a case report. Int J Surg Case Rep. 2021;82:1-4.

6. Alekseyev K, Didenko L, Chaudhry B. Rhinocerebral mucormycosis and COVID-19 pneumonia. J Med Cases. 2021;12(3):85-9. doi: 10.14740/jmc3637.

7. McDonald PJ. Author, Chandrasekar PH chief Editor. Mucormycosis (Zygomycosis) [Internet]. Medscape; 2018. p. 10.

8. Covid A. 19 Pandemic, India reports over 11,700 black fungus cases Check state-wise report on Mucormycosis cases here. Jagran Engl; 2021.

9. Coronavirus India Tracker: state-wise Covid-19 cases. Decanherald. Available from: https://www.deccanherald.com/national/coronavirus-indiatracker-state-wise-covid-19-cases-deaths-on-may-25989686.html. [Last accessed on 25 May 2021]

10. Raut A, Huy NT. Rising incidence of mucormycosis in patients with COVID-19: another challenge for India amidst the second wave? Lancet Respir Med. 2021 Jun;9(8). doi: 10.1016/S22132600(21)00265-4.

11. Prakash H, Chakrabarti A. Epidemiology of mucormycosis in India. Microorganisms. 2021;9(3):523. doi: 10.3390/microorganisms9030523.

12. Evidence-based advisory in the time of COVID-19 (screening, diagnosis and management of mucormycosis); 2021. Available from:

https://www.icmr.gov.in/pdf/covid/techdoc/Mucormycosis_A DVISORY_FROM_ICMR_In_COVID19_time.pdf. [Last accessed on 05 Jun 2021]

13. Zakaria A, Osman M, Dabboussi F, Rafei R, Mallat H, Papon N, Bouchara JP, Hamze M. Recent trends in the epidemiology, diagnosis, treatment, and mechanisms of resistance in clinical Aspergillus species: A general review with a special focus on the Middle Eastern and North African region. J Infect Public Health. 2020;13(1):1-10. doi: 10.1016/j.jiph.2019.08.007.

14. Koehler P, Bassetti M, Chakrabarti A, Chen SCA, Colombo AL, Hoenigl M, Klimko N, Lass-Flörl C, Oladele RO, Vinh DC, Zhu L, Boll B, Bruggemann R, Gangneux J, Perfect JR, Patterson TF, Persigehl T, Meis JF, Ostrosky-Zeichner L, White PL, Verweij PE, Cornely OA. Defining and managing COVID-19-associated pulmonary aspergillosis: the 2020 ECMM/ISHAM consensus criteria for research and clinical guidance. Lancet Infect Dis. 2021;21(6):e149-62. doi: 10.1016/S1473-3099(20)30847-1.

15. Mohamed A, Rogers TR, Talento AF. COVID-19 associated invasive pulmonary aspergillosis: diagnostic and therapeutic challenges. JoF. 2020;6(3):115. doi: 10.3390/jof6030115.

16. Arastehfar A, Carvalho A, van de Veerdonk FL, Jenks JD, Koehler P, Krause R, Cornely OA, Perlin S. COVID-19 associated pulmonary aspergillosis (CAPA)-from immunology to treatment. J Fungi 2020;6:91.

17. Riad A, Gomaa E, Hockova B, Klugar M. Oral candidiasis of COVID-19 patients: case report and review of the evidence. J Cosmet Dermatol. 2020;20:1580-4.

18. Arya NR, Naureen BR. Candidiasis [internet]; 2021. Stat Pearls. Available from: https: candidiasis-statpearls-NCBIbookshelf (NIH.gov). [Last accessed on 16 Jun 2021]
19. Isalkar U. Don't call Candida-linked infections white fungus. The times of India: Forthcoming; 2021.

20. Pfaller MA. Epidemiology of candidiasis. J Hosp Infect. 1995;30:329-38. doi: 10.1016/0195-6701(95)90036-5.

21. Fu J, Ding Y, Wei B, Wang $\mathrm{L}, \mathrm{Xu} \mathrm{S}$, Qin $\mathrm{P}$, Wei L, Jiang $\mathrm{L}$. Epidemiology of Candida albicans and non-C. albicans of neonatal candidemia at a tertiary care hospital in western China. BMC Infect Dis. 2017;17(1):329. doi: 10.1186/s12879-017-2423-8.

22. Traci C, Johnson MD. What is candidiasis? [internet]; 2020. WebMD. Available from: https://www.webmd.com/skinproblems-and-treatments/guide/what-is-candidiasis-yeastinfection\#2-6. [Last accessed on 23 Jun 2021]

23. Rajendra Santosh ABJ, Muddana K, Bakki SR. Fungal infections of oral cavity: diagnosis, management, and association with COVID-19. SN Compr Clin Med 2021;3(6):1373-84. doi: 10.1007/s42399-021-00873-9.

24. Abohelwa MM, Rio-Pertuz GD, Parmar KN, Morataya C, Siddique S, Duangkham S, Nugent KM. Pulmonary cryptococcosis in the 2019 novel coronavirus, when the coinfection affects the mortality. Am J Respir Crit Care Med. 2021;203:2461.

25. Bhattacharya S, Banerjee A. Cryptococcosis in an advance state of aids and its challenging therapeutic regimen: is it really curable? Asian J Pharm Clin Res. 2015;8:51-3.

26. Outlookindia. Available from: https://www.outlookindia. [Last accessed on 24 Jun 2021]

27. Hughes S, Troise O. Oral candidiasis: causes, types, and treatment. Pharm J. 2020 Nov;305(7943).

28. White Fungus. Available from: https://www.drishtiias.com/daily-updates/daily-newsanalysis/white-fungus. [Last accessed on 24 Jun 2021]

29. Why are the fungal infections named after colours-Black, white and yellow? Read here. Available from: https://www.mumbailive. [Last accessed on 24 Jun 2021]

30. Decoding the Mysterious yellow fungus; experts call for genetic testing; c2021. Available from: https://www.in/news/health/2021/05/25/decoding-themysterious-yellow-fungus-experts-call-for-genetictesting.html. [Last accessed on 01 Sep 2021]

31. Randhawa HS, Kowshik T, Preeti Sinha K, Chowdhary A, Khan ZU, Yan Z, Xu J, Kumar A. Distribution of Cryptococcus gattii and Cryptococcus neoformans in decayed trunk wood of Syzygium cumini trees in north-western India. Med Mycol. 2006;44(7):623-30. doi: 10.1080/13693780600860946.

32. Alchetron.com [Internet]. Aspergillus fumigatus; c2018. Available from: https://alchetron. com/Aspergillus-fumigatus. [Last accessed on 25 Jun 2021]

33. Wionews.com [Internet]. India's first case of 'green fungus' infection reported in Madhya Pradesh: All you need to know; c2021. Available from: https://www.wionews.com/indianews/indias-first-case-of-green-fungus-infection-reported-inmadhya-pradesh-all-you-need-to-know-391837. [Last accessed on 24 Jun 2021].

34. Indiatimes.com [Internet]. What is Green fungus: key things to know; c2021. Available from: https://timesofindia.indiatimes.com/india/what-is-greenfungus-symptoms-prevention-and-key-things-toknow/articleshow/83600263.cms\#: :text=Why\%20is\%20it\%2 0called\%20Green,medicine\%20HoD\%20Dr\%20Ravi\%20Dosi. [Last accessed on 24 Jun 2021]. 